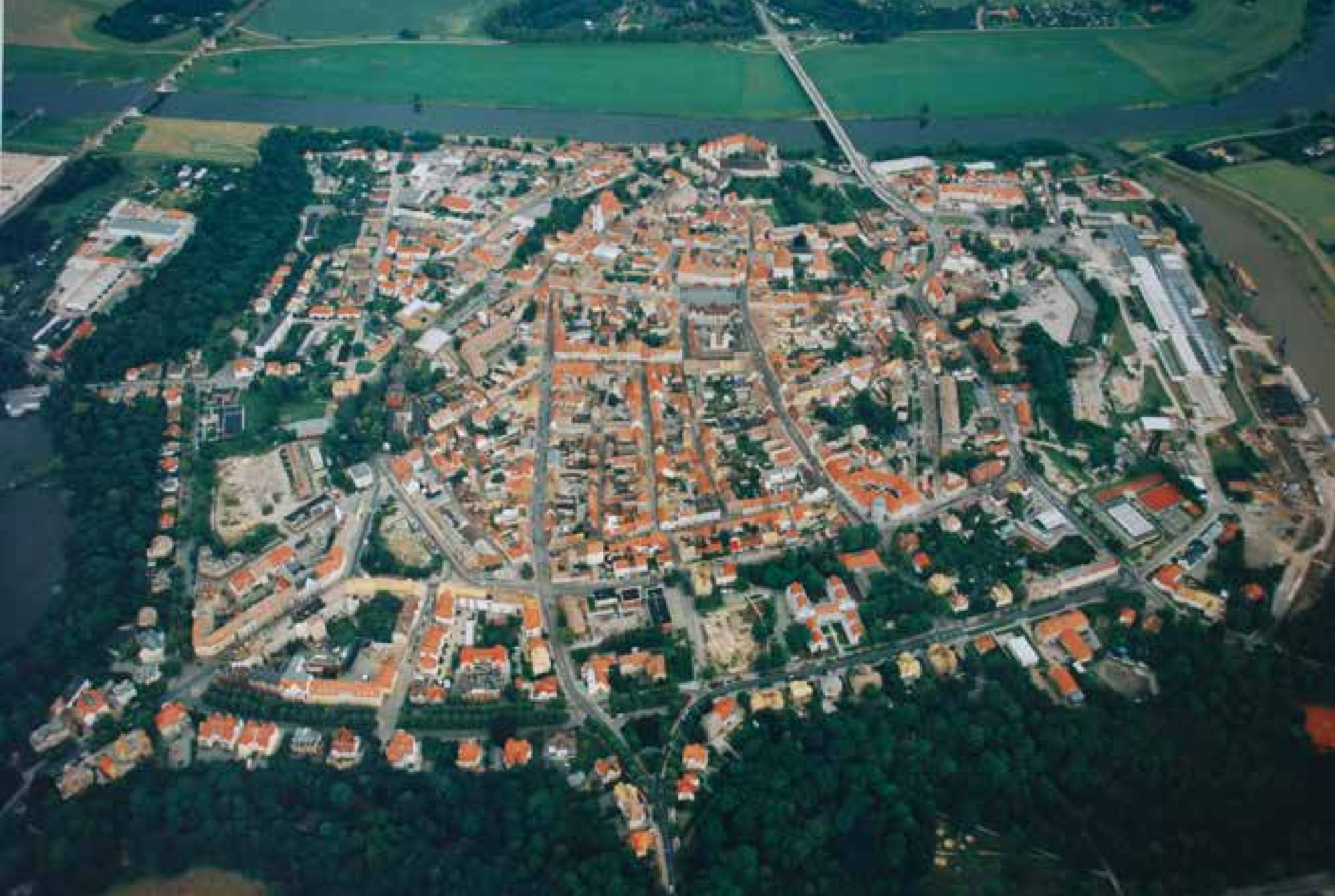

\title{
Die Bierstadt Torgau und ihre Bierkeller
}

\author{
Jürgen Herzog
}

Die Renaissance- und Reformationsstadt Torgau erlebte vom Ende des 15. Jahrhunderts bis zum Dreißigjährigen Krieg, also in der Zeit des sogenannten langen 16. Jahrhunderts, eine wirtschaftliche und kulturelle Blüte, die noch heute an der Architektur ihrer Altstadt ablesbar ist. Dazu trug in besonderem Maße die herrschaftliche Hofhaltung der sächsischen Kurfürsten bis 1550 als Hauptresidenz $^{1}$ und danach als Nebenresidenz, Landtagsstadt und „heimliche Hauptstadt“, wie sie jüngst bezeichnet worden ist, bei. ${ }^{2}$ Einwohnerzuwachs, prosperierende Wirtschaft und überregionaler Handel förderten darüber hinaus den Wohlstand der Bürgerstadt maßgeblich.

Die Stadt des 16. Jahrhunderts war im Wesentlichen eine steinerne, neue Stadt. Ein totaler Stadtbrand 1442 hatte die mittelalterliche Bebauung vollständig, ein weiterer nach Wiederaufbau 1482 nochmals zu einem Drittel zerstört. ${ }^{3}$ Erhalten blieb die unterirdische Bebauung, die Keller, die sich heute in der Regel durch einen schwarzen Belag von den jüngeren aus der Zeit nach den Bränden unterscheiden.

Hauptproduktionszweig und Haupthandelsgut war das Torgauer Bier, das zeitgenössisch als das „Brot der Stadt“ gegolten hat. Zur Bestätigung der Privilegien der Stadt während der Huldigung der Brüder Kurfürst Ernst und Herzog Albrecht hieß es 1464: „und Confirmirten selbiger Stadt ihre Privilegien, insonderheit das brauen alda, als der Stadt beste Nahrung." ${ }^{4}$

Torgauer Bier war ein deutschlandweit berühmtes Spitzenbier, das auch als Festtagsbier galt. Der Arzt Dr. Heinrich Knaust beurteilte 1575 die Qualität nach angeblich eigener Verkostung aller beschriebenen Biere: „Torgisch Bier. Das Land zu Meichsen / gibt keinem Land nichts zuvor / an der Zal / Mannichfeltigkeit / Güte vnd Furtreffligkeit der Biere [...] Vnd ist unter diesen Bieren das Torgisch Bier / fast (sehr) wol bekandt / vnd mich
Luftaufnahme der

Torgauer Altstadt

- Stadtverwaltung Torgau

1 Jürgen Herzog: Vorreformatorische Kirche und Reformation in Torgau. Beucha/ Markleeberg 2017, S. 230.

2 Christian Heinker: Kontrollieren oder Delegieren? Zur Interaktion Kurfürst Augusts mit seinen geheimen Räten. In: Winfried Müller/Martina Schattkowsky/Dirk Syndram (Hrsg.): Kurfürst August von Sachsen. Beiträge zur wissenschaftlichen Tagung vom 09 . bis 11 . Juni in Torgau und Dresden. Dresden 2017 S. 102. 
Anzahl von Gebräuden und Brauberechtigungen sächsischer Städte gedüncket / das es ein trefflich Bier sey / nicht allein an der Stärke / sondern auch an der Güte / denn es hat einen guten Geschmack / vnd gibt gut Nutriment, denn aliment [Nahrungsmittel] vnd Nahrung / auch Krafft den vornembsten Gliedern am Menschen / denn es hat einen Geschmack vnd Geruch / nicht anders / als were diß Bier gewürtzet.“5 Martin Luther pries es: „Cerevisiana torgama quam libenter velle esse vinum“ [Torgauer Bier ist viel besser als Wein]. ${ }^{6}$

Nach gegenwärtigem Forschungsstand lässt sich nach der Anzahl der Gebräude, der sogenannten Biere, und der Brauberechtigungen schließen, dass Torgau im 16. Jahrhundert die größte Bierbraustadt in Sachsen gewesen ist. ${ }^{7}$

\begin{tabular}{|lcc|}
\hline & Gebräude & Berechtigte \\
Chemnitz (1513) & & 175 \\
Dresden (1626) & 1.226 & 212 \\
Eilenburg (um 1630) & 780 & \\
Freiberg (1577) & 762 & $141-143$ \\
Grimma (1660) & & 371 \\
Leipzig (1530) & 862 & 268 \\
Torgau (1524) & 1.475 & 172 \\
Wittenberg (1513) & & \\
Wurzen (1543) & ca. 540 & 243 \\
Zwickau (1525) & 858 & \\
\hline
\end{tabular}

3 Michael Böhme: Chronik von Torgau, Universitätsund Landesbibliothek Sachsen-Anhalt, Hist. $2442^{\circ}$, S. 143 und S. 155.

4 Stadtarchiv Torgau (im Folgenden: StAT), H 577, Statuten und Willkühr 1557, 2r.

5 Heinricum Knaustium: Fünff Bücher von der Göttlichen und Edlen Gabe [...] Bier zu brauen. Erfurt 1614, ohne Seitenzahlen, unveränderte Ausgabe von 1575.

6 Martin Luther: D. Martin Luthers Werke. Weimarer Ausgabe. Abteilung 3. Briefwechsel. Bd. 3. Briefe 15231525. Weimar 1933, S. 539, Nr. 898.

7 Jürgen Herzog: Beiträge zur Residenz- und Reformationsgeschichte der Stadt Torgau. Beucha 2008, S. 58-59.

8 Karlheinz Blaschke: Die geschichtliche Entwicklung der Stadt Torgau von den Anfängen bis zum Beginn des 19. Jahrhunderts. In: Peter Findeisen/Heinrich Magirius: Die Denkmale der Stadt Torgau. Leipzig 1976, S. 19.

Höchstanzahl der Gebräude (Biere) auf den Grundstücken der Stadt, um 1520, eingetragen in den heutigen Stadtgrundriss Bearbeitung: Jürgen Herzog

\section{Braurechte}

Braurechte gehören zu den ältesten und wichtigsten Vorrechten der Stadtbürger, deren Vergabe „erfahrungsgemäß in die Zeit unmittelbar nach der Stadtgründung“ zurückreicht, also für Torgau in die Zeit um $1200 .^{8}$

Aufgrund von Tranksteuerlisten lässt sich die Gebräudeanzahl für die Zeit um 1520 im heutigen, über Jahrhunderte unveränderten Stadtplan darstellen. Wenn man ferner davon ausgeht, dass wesentliche Veränderungen seit ihrer Vergabe nicht erfolgt sind, lassen sich Schlussfolgerungen zur Stadtentstehung ableiten.

Kennzeichnend ist, dass die Braurechte der Bürger auf eine ursprüngliche Beschränkung auf die Hauptgassen der planmäßig angelegten Bürgerstadt hinweisen. Der herrschaftlich und geistlich bestimmte Stadtteil zwischen Schloss und Markt, der weitestgehend nicht unter Stadtrecht stand, verfügte ursprünglich über keine Braurechte.

Dass darüber hinaus die Anzahl der Gebräude in einer Berechtigungsspanne von einem bis zu zwölf von der Grundstücksgröße abhängig war, kann als Bestätigung der Ursprungssituation angesehen werden. ${ }^{9}$ Es hat sich dabei um eine ursprünglich hoheitliche Privilegierung gehandelt, die mit der Entrichtung eines Bierzinses verbunden war, wie sich aus einer Stiftung Markgraf Wilhelms I. von Meißen aus dem Jahr 1386 ergibt. In ihr wird der „birzins“ einem Jacobsaltar in der Marktkirche St. Nikolai der Stadt übereignet. ${ }^{10}$ Er findet sich im 16. Jahrhundert als so genannter Rauchzins wieder. ${ }^{11}$ Eine mittelalterliche Bodenprobe aus einer Kulturschicht in der Torgauer Bäckerstraße, die in das 13./14. Jahrhundert datiert ist und archäobotanischer Makrorestuntersuchung unterzogen wurde, bestätigt durch einen hohen Anteil an Hopfen und schwarzem Bilsenkraut frühe Brautätigkeit. ${ }^{12}$

Das Braurecht in der Stadt Torgau war, wie auch in anderen Städten, wenn von Dienst- und Gnadenbieren abgesehen wird, grundsätzlich ein Realrecht auf bürgerlichen Grundstücken, den so genannten Brauerben. Dabei wurde der Begriff Brauerbe sowohl für das brauberechtigte Grundstück als auch für den Inhaber, den Brauherren, gebraucht. Abtrennungen und Verkauf von Brau-

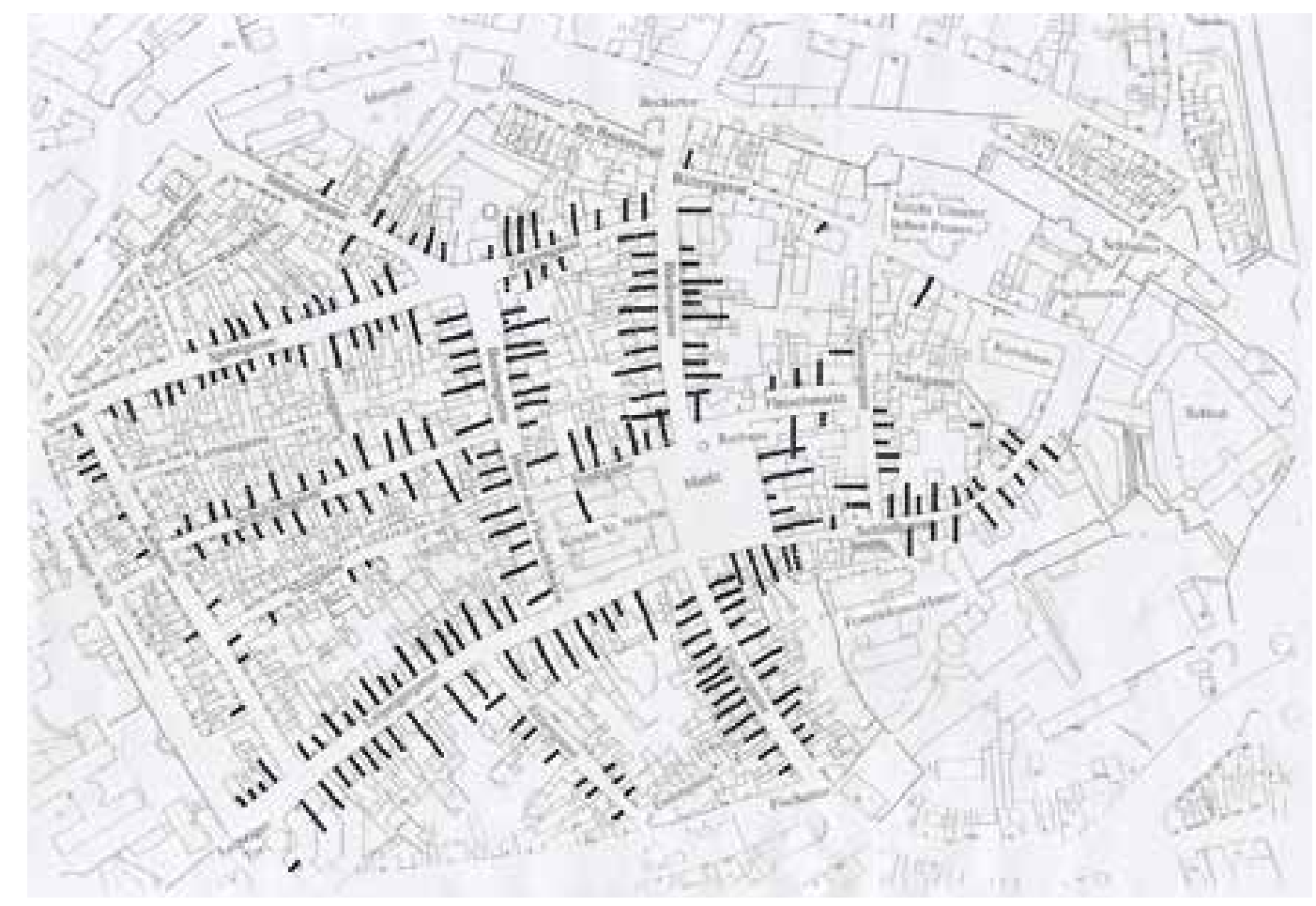


rechten sind in der Regel unterbunden worden. Vorstadtgrundstücke hatten keine Braurechte. Dienstbiere durften nur für den eigenen Bedarf gebraut werden und waren an die Ausübung eines Dienstverhältnisses, z. B. als Pfarrherr gebunden. ${ }^{13}$ Gnadenbiere wurden vorwiegend während der Torgauer Residenzzeit durch den Kurfürsten seinen Hofdienern und Freihausbesitzern für den Eigenbedarf gewährt und waren ein ständiges Ärgernis für den Torgauer Rat.

\section{Brauordnung}

Schriftlich gefasste Brauordnungen waren spätestens seit dem 15. Jahrhundert vorhanden, die älteste überlieferte stammt jedoch erst aus dem Jahr 1557. Die erste gedruckte Ordnung liegt aus dem Jahr 1622 vor. ${ }^{14}$ Danach war u. a. geregelt:

- brauen durften nur Bürger, die ein Brauerbe besaßen und den Braueid geschworen hatten,

- die Brauherren durften nur in ihren eigenen Häusern Bier ausschenken,

- fremdes Bier war in der Stadt nicht zugelassen,

- für ein Gebräude mit acht Fass durften nicht mehr als 32 Torgauer Scheffel Gerste begossen werden,

- die Braudurchführung lag allein in den Händen der vom Rat vereidigten Braumeister ${ }^{15}$

Die Einhaltung der Brauordnung stand unter strenger Kontrolle des Rates. Damit konnte lange Zeit eine hohe Qualität des begehrten Torgauer Biers gesichert werden.

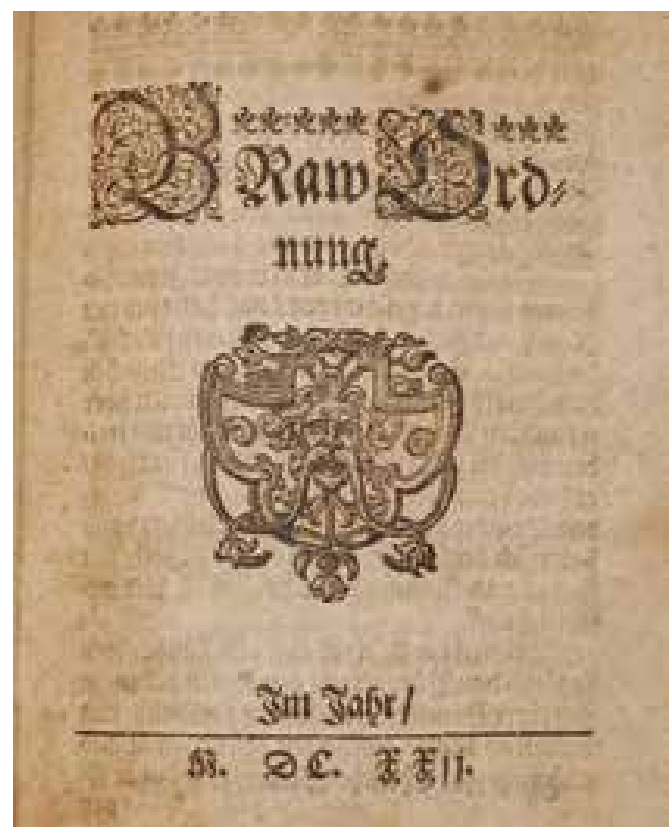

\section{Braudurchführung}

Die Brautätigkeit war aus technologischen Gründen Saisonarbeit während der kalten Jahreszeit und wurde in der Regel von Michaelis (29. September) bis Ostern durchgeführt. Der Torgauer Rat ließ den jeweils noch in der Stadt vorhandenen Biervorrat durch den Stadtschreiber auszählen und legte dann den Braubeginn fest. Den Brauherren wurden Brauzeichen ausgehändigt, die den

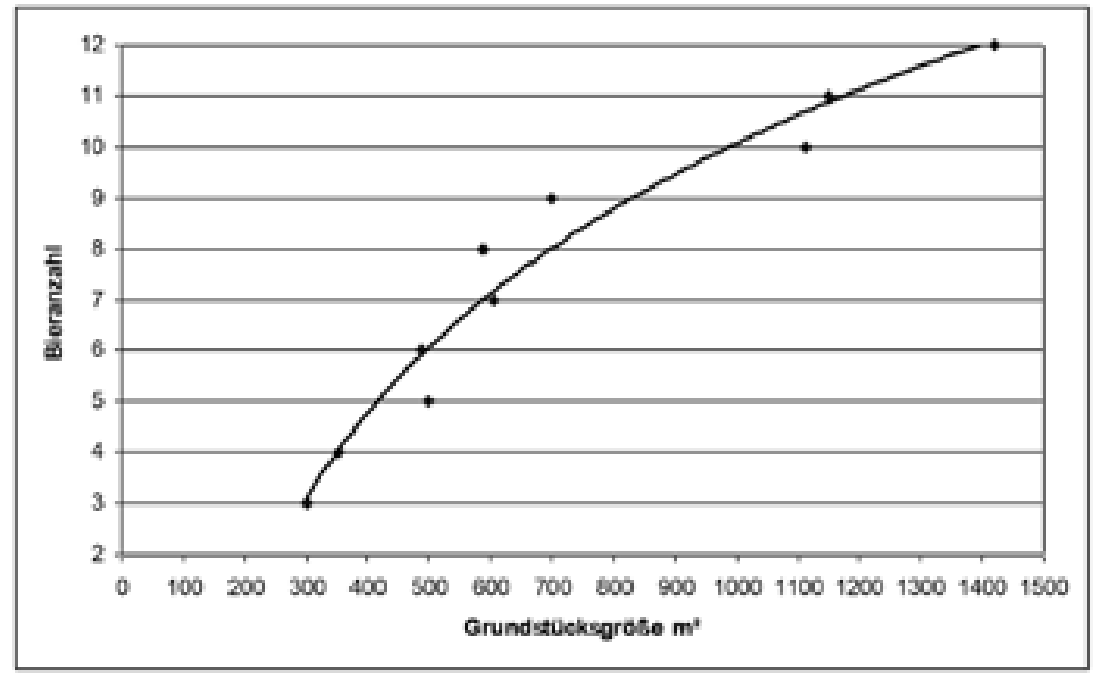

Braumeistern zur Kontrolle zu übergeben waren. Eine zeitgenössische Beschreibung des Brauvorgangs für Torgauer Bier liegt nicht vor. Heinrich Knaust hat den üblichen Brauvorgang 1575 stark verkürzt so beschrieben: „Man nimpt das Korn / Gersten / oder Weizen / So nun eingewassert / wider getrucknet / gedurret / vnd in der Mühlen fein grob gemahlen ist / vnd kocht es eine Zeitlang / insonderheit mit Wasser / oder geust heiß Wasser daruber her. Darnach last man es durchlauffen / vnd seuget es / folgends thut man den Hopffen hinein / vnd seudt es damit / biß so lange / daß der Hopffe seine Bitterkeit zum theil verleurt. Hernach lest man diß gekochte abermals / vnd noch eins durchlauffen. Vnd als dann ists Bier / So last mans denn hernach ziemlich kalt werden / vnd darnach gibt man ihm die Hefen / daß es dadurch erwarme / vnd wenn denn solches abermal auch geschehen ist / so fasset man es / vnd bringet oder thue es in die Bierfässer / vnd ist zwar kein Zweifel daran / das Bier neme an sich eine warme Art vnd Natur / durch jetzt gemeldetes Kochen vnd Brawen." ${ }^{16}$

Zuerst braute man Schankbier, auch junges Bier genannt, nach einer weiteren Entscheidung des
Abhängigkeit der Braurechte von der Grundstücksgröße Bearbeitung: Jürgen Herzog
Braw Ordnung im Jahr / MDCXXii (1622)

Foto: Jürgen Herzog

Torgauer Brauzeichen des 16. Jahrhunderts im Stadt- und Kulturgeschichtlichen Museum Torgau Foto: Jürgen Herzog

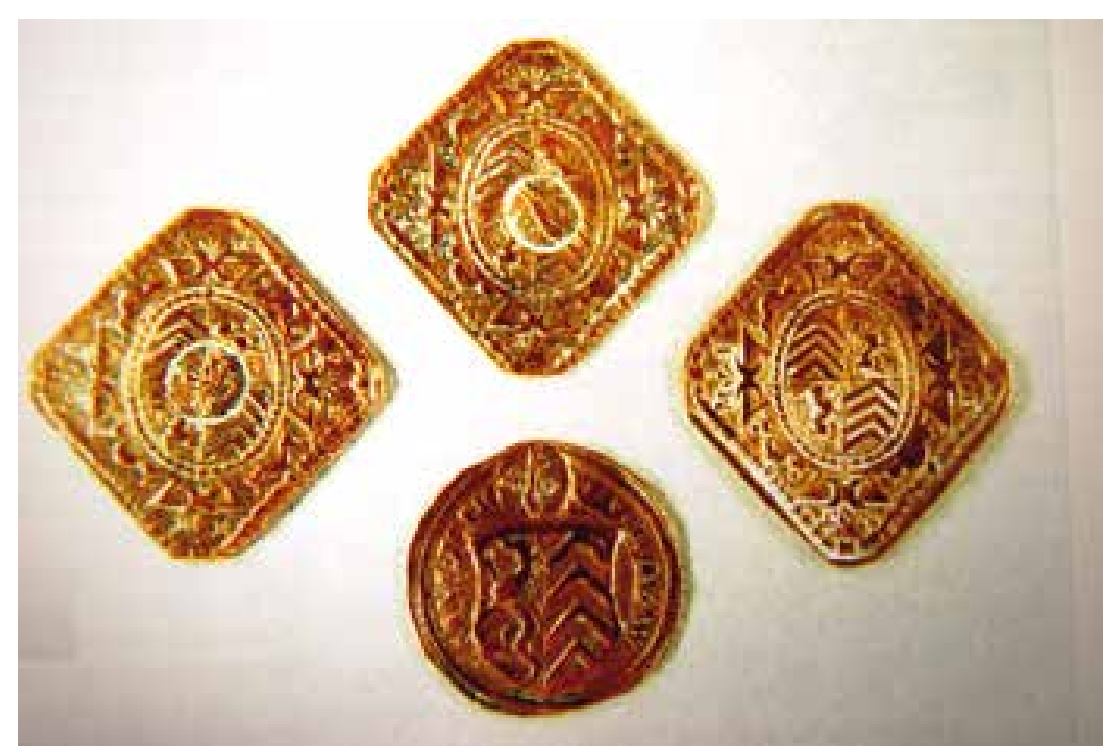


9 Jürgen Herzog: Braurechte und Bierproduktion am Anfang des 16. Jahrhunderts in der Stadt Torgau. In: Neues Archiv für sächsische Geschichte 84 (2013), S. 23-24.

10 Carl Knabe: Urkundenbuch von Torgau. Torgau 1902, S. 19, Nr. 43.

11 StAT, H 2735, Rechnung des Gemeinen Kastens 1529, unpaginiert.

12 Christoph Herbig: Archäobotanische Großrestuntersuchungen in der Altstadt von Torgau: Grabung TG-127. In: Arbeits- und Forschungsberichte zur sächsischen Bodendenkmalpflege, Beiheft 31. Dresden 2017, S. 336337.

13 Carl Knabe: Die Torgauer Visitationsordnung von 1529 . Torgau 1881, S. 4.

14 StAT, B 209 a, Des Raths zu Torgau [...] Statuta 1622.

15 StAT, H 576, Privilegien und Statuten, 1557, 42r-59r.

16 Knaustium 1616 (wie Anm. 5).

17 Sächsisches Staatsarchiv, Hauptstaatsarchiv Dresden, 10036 Finanzarchiv, Rep. A 25a I, I Nr. 2343, Torgauer Amtsinventar 1610, unpaginiert.

18 Herzog 2008 (wie Anm. 7), S. 58-59.

Rates, in der Regel ab Ende November oder Anfang Dezember, Lagerbier. Die Brautätigkeit erfolgte in privaten Brauhäusern; kommunale Brauhäuser gab es in Torgau nicht.

Außer bei den Brauerben und den sonderberechtigten Einwohnern wurde in Torgau auch am kurfürstlichen Hof gebraut. Dafür standen ein Gießund Malzhaus und ein Brauhaus, unmittelbar vor dem Schloss gelegen, zur Verfügung. ${ }^{17}$ Die Inanspruchnahme städtischer Torgauer Braumeister für die Braudurchführung des Hofes ist erstmalig für 1397 nachgewiesen. ${ }^{18}$

\section{Bierproduktion}

Die Produktionsmenge ist auf Grund der Quellenlage für den betrachteten Zeitraum nicht durchgängig ermittelbar, besser jedoch die Anzahl der Gebräude.

Da die Gesamtanzahl der verfügbaren Gebräude im Interesse der Brauherrschaften, zu denen immer der Rat und die Viertelsmeister gehörten, grundsätzlich nicht erhöht worden ist, um Überproduktion und Preisverfall auszuschließen, haben sich die Produktionsmengen nicht grundsätzlich verändert. Ein begrenzter Anstieg erfolgte in der ersten Hälfte des 16. Jahrhunderts während der Torgauer Residenzzeit und an der Wende zum 17. Jahrhundert.

In der Brauzeit 1514/15 wurden 8.051 Fass produziert, $1523 / 24$ waren es 8.372 Fass. Bis 1534 stieg die Jahresproduktion auf 10.740 und 1545 auf 11.210 Fass. 1596 lag sie bei 11.376 Fass. ${ }^{19}$ Damit wurden Höchstwerte der Starkbierproduktion erreicht. Da der Fassinhalt bei 335 Litern gelegen hat, entspricht das einer Jahresproduktion von etwa 3,8 Millionen Litern. ${ }^{20}$ In dieser Menge ist die Produktion von Nachbieren, dem so genannten Sanftbier und dem Kofent, nicht enthalten.

\section{Brauhäuser}

Entwicklung der Gebräudeanzahl Bearbeitung: Jürgen Herzog
Während des 16. Jahrhunderts verfügten etwa 220 Brauerbengrundstücke über eigene Brauhäuser,

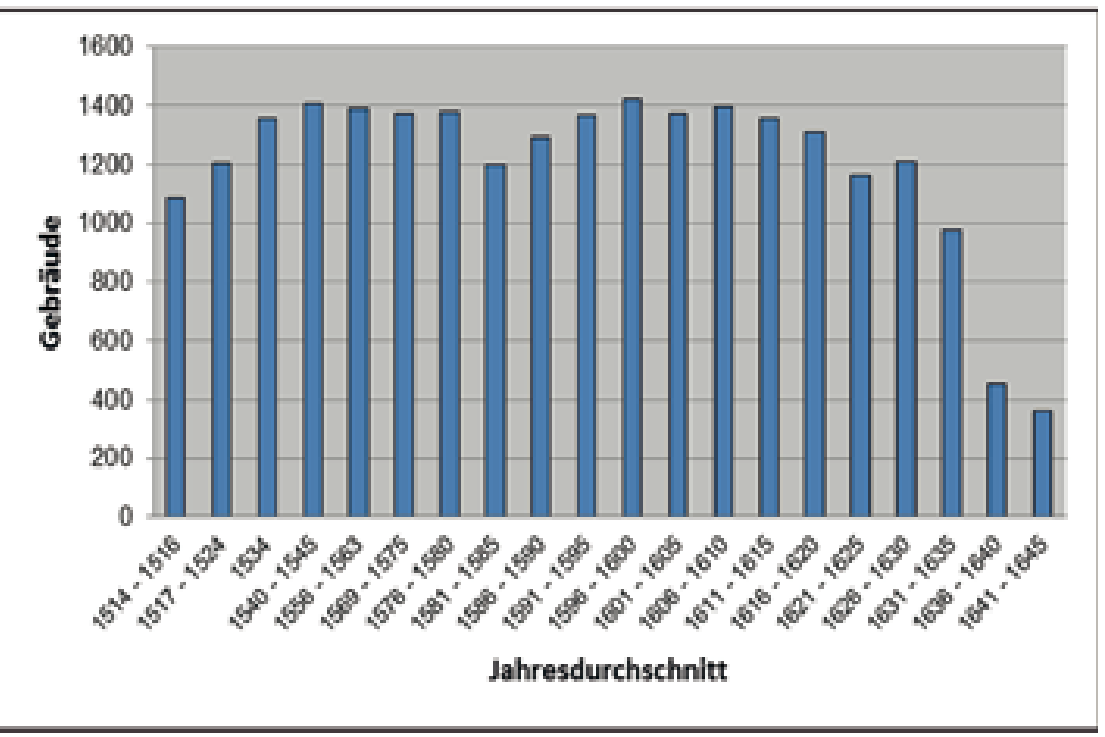

die zugleich als Malzhäuser genutzt worden sind. Grundstücke mit sechs oder mehr Gebräuden hatten immer ein eigenes Brauhaus, die mit weniger als drei Gebräuden dagegen keines, so dass auch auf Mietbasis in fremden Brauhäusern gebraut werden musste.

Nur wenige Brauhäuser sind heute noch vorhanden. Einige wurden zu Wohn- und Gewerbezwecken umgebaut, weitere sind noch durch Abbruch der Obergeschosse als Abstellschuppen vorhanden. Häufig befindet sich die ursprüngliche Nutzung weder im allgemeinen noch im denkmalpflegerischen Bewusstsein.

Ein großes und das am besten erhaltene Brauhaus befindet sich auf dem heutigen Grundstück Fischerstraße 11 und wird mit seinen Braukellern als Braumuseum im Rahmen des Torgauer Museumspfades betrieben. Es gehörte zu einem Brauerbe mit der Brauberechtigung von elf Gebräuden. Das Brauhaus wurde als Massivbau in Ziegeln an Stelle eines Vorgängerfachwerkbaus um 1565 errichtet und verfügt über sieben Geschosse: ein Kellergeschoss, Erdgeschoss, zwei Obergeschosse und drei Dachgeschosse. Die Sudhalle im Erdgeschoss besteht aus einer dreischiffigen kreuzgratgewölbten Halle mit zwei Sandsteinmittelpfeilern und hat eine Geschossfläche von 106 Quadratmetern. Die Beschickung vom darüber liegenden Malzboden erfolgte durch Gewölbeöffnungen.

Nach dem Sudvorgang wurde das frische Bier durch Öffnungen im Fußbodenbereich in die Fässer im Gär- und Lagerkeller abgelassen. Dafür standen im Kellergeschoss zwei Kellergewölbe mit einer Grundfläche von 32 Quadratmetern und 40 Quadratmetern zur Verfügung. Diese Gewölbe sind vollständig in Sandstein ausgeführt, einem teuren Baumaterial. Diese Art der Sandsteinausführung ist in Torgau seit dem 16. Jahrhundert üblich. Der poröse diffusionsoffene Sandstein war besonders dafür geeignet, günstige klimatische Bedingungen für eine längere Bierlagerung zu gewährleisten. Eine längere Lagerfähigkeit des Torgauer Biers gehörte zu seinen Vorzügen.

Unter dem Vorderhaus, dem Wohnhaus Fischerstraße 11, sind die mittelalterlichen Keller erhalten. Der Zugang zu den Kellern erfolgte, wie es bei mittelalterlichen Häusern häufig war, vom Hof aus. Auf der Kellertreppe gibt es einen gesonderten vierstufigen Abgang aus der Küche im Erdgeschoss. Damit war ein kurzer Weg zum Küchenkeller vorhanden.

Die Unterkellerung unter dem Wohnhaus ist zweigeschossig. Ein unteres Kellergeschoss ist in den anstehenden Felsen eingetieft. Zur Straße gelegen befindet sich ein aus Natursteinen gemauertes Gewölbe mit Gewölbescheitel in Nord-/Südlage, das in Lehm verfugt ist. Die verwendeten Porphyrbruchsteine bestehen aus demselben Material, wie der anstehende Felsen und sind aus ihm gewonnen. Der Felsen und die Tonne haben einen schwarzen Belag. Es ist vorstellbar, dass dieser Keller dem 13. oder 14. Jahrhundert zuzuordnen ist. Er hat eine Grundfläche von 37 Quadratme- 


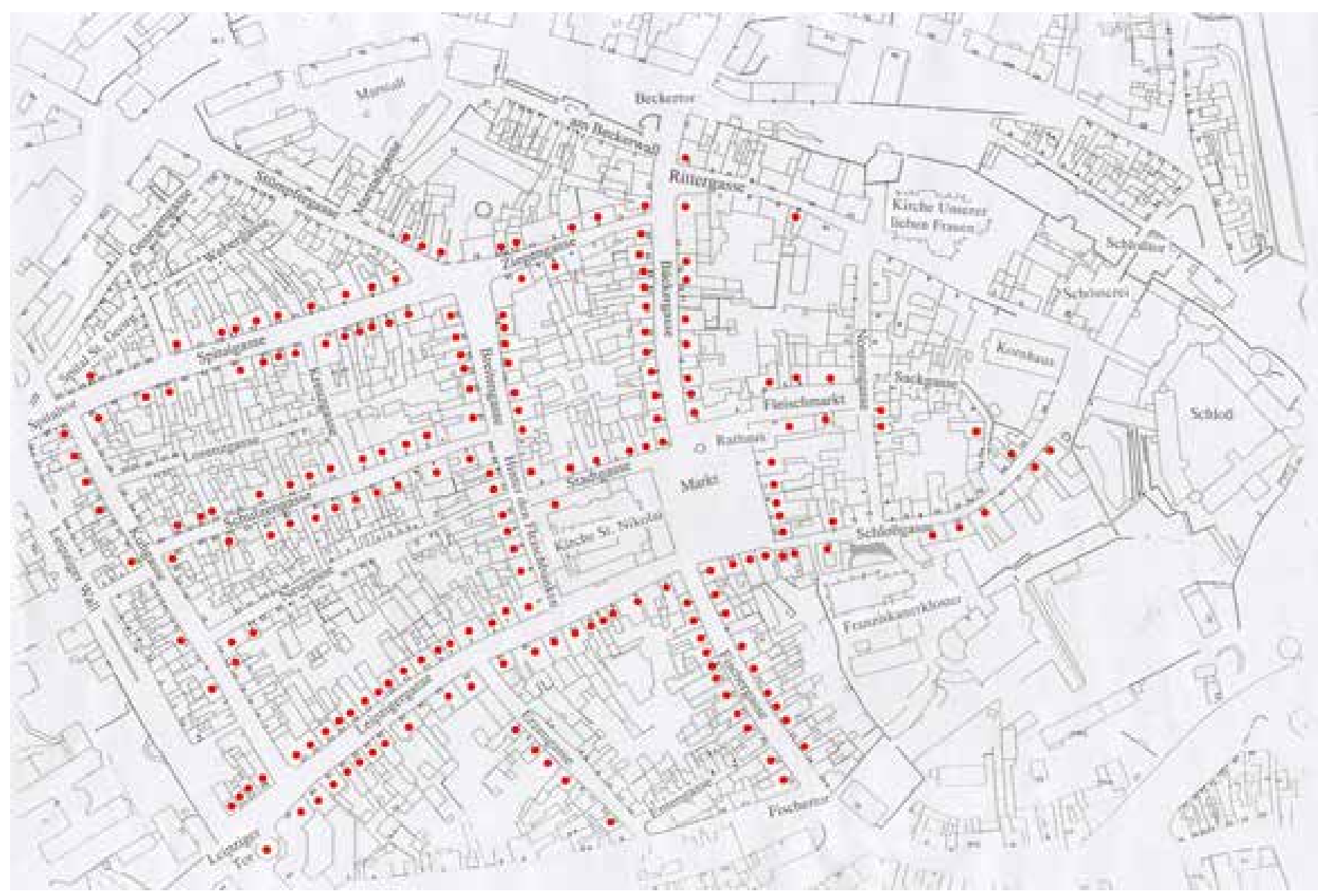

tern. Westlich hinter dieser Tonne liegt eine zweite Kellertonne in Mischmauerwerk-Bruchstein, Sandstein und Ziegel, die einer mittelalterlichen Hauserweiterung vor 1482 zuzuordnen ist, und damit der Zeit vor dem letzten Stadtbrand in dieser Straße.
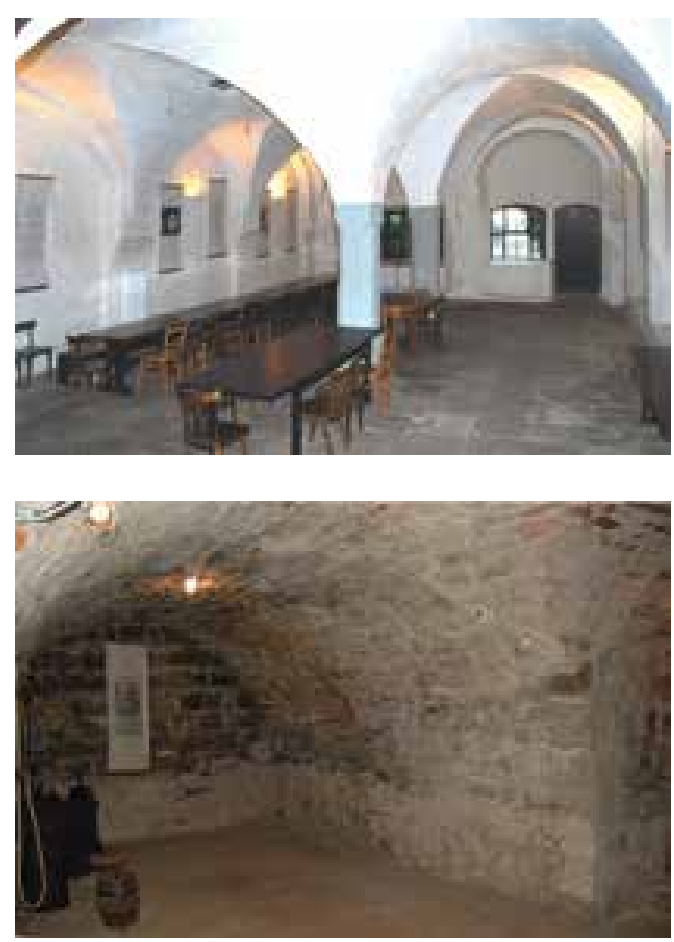

Ein darüber liegendes oberes Kellergeschoss steht mit einer Niveauerhöhung des Straßenbereichs, auch den Bränden geschuldet, in Verbindung. Eine kreuzgratgewölbte, flache Tonne (28 Quadratmeter) vorwiegend in Ziegeln gesetzt und ein kleiner, der vermutliche Küchenkeller (14 Quadratmeter)
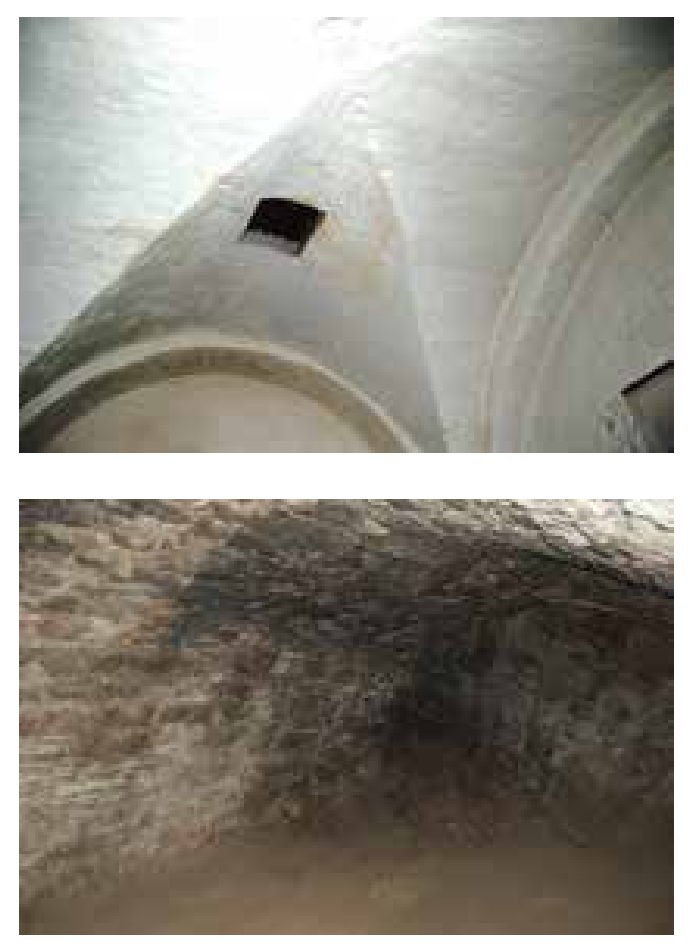

Brauerbengrundstücke mit eigenen Brauhäusern um 1520, eingetragen in den heutigen Stadtgrundriss

Bearbeitung: Jürgen Herzog

links: Brauhaus Fischerstraße 11, Sudhalle

Foto: Jürgen Herzog

rechts: Brauhaus Fischerstraße 11, Gewölbeöffnung von der Sudhalle zum Obergeschoss

Foto: Jürgen Herzog

links: Brauhaus Fischerstraße 11, vorderes Kellergewölbe unter dem Sudhaus

Foto: Jürgen Herzog

rechts: Brauhaus Fischerstraße 11, unteres Kellergewölbe unter dem Wohnhaus

Foto: Jürgen Herzog 
Keller Leipziger Straße 34, Bierablassröhre im Gewölbescheitel der Kellertonne, Foto: Jürgen Herzog

19 Thüringisches Hauptstaatsarchiv Weimar, Ernestinisches Gesamtarchiv, Reg. Qq Abt. B 3928-3930 und 39453947, Tranksteuerregister der Stadt Torgau 1534 und 1545; Jürgen Herzog: Torgauer Bier und der Leipziger Burgkeller. Beucha/Markkleeberg 2015, S. 36.

20 Herzog 2015 (wie Anm. 19), S. 41.

21 Carl Knabe: Torgauer Namenbuch bis 1535, Handschrift 1911; StAT H 646, S. 140.

22 Findeisen/Magirius 1976 (wie Anm. 8).

Kellerstruktur Torgauer Grundstücke in Abhängigkeit von der Gebräudeanzahl, Anzahl der erfassten Keller in Klammern

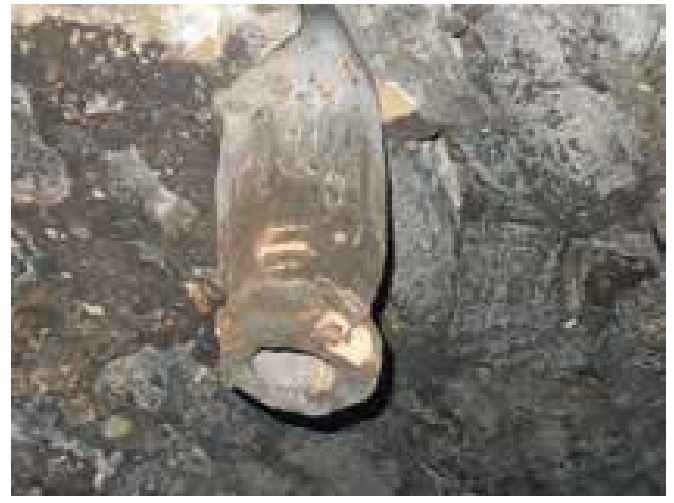

liegen auf gleicher Ebene und dürften nach dem Brand von 1482 entstanden sein. Der damalige Besitzer, der Schuhmacher Kirsten Schlope, kaufte 1483 für den Neuaufbau Kalk, Steine und Ziegel. ${ }^{21}$ Auf eine Besonderheit sei noch hingewiesen: In einigen Kellern befinden sich in die Gewölbe eingelassene hölzerne Bierablassröhren anstatt sonst üblicher einfacher Öffnungen im Gewölbescheitel. Bisher wurden unterschiedliche Längen bis zu 135 $\mathrm{cm}$, Außendurchmesser von etwa $15 \mathrm{~cm}$ und Innendurchmesser von etwa $6 \mathrm{~cm}$ gemessen. Ein Beispiel dafür ist im mittelalterlichen Keller des Hauses Leipziger Straße 34 erhalten, das über fünf Gebräude verfügt hat.

In einer ersten orientierenden Bestandsaufnahme im Jahr 2014 wurden 70 Keller erfasst und einer Auswertung unterzogen. zahl der Gebräude zwischen durchschnittlich 24,6 Quadratmetern (drei Gebräude) und 63,6 Quadratmetern (zehn bis zwölf Gebräude).

Die Küchenkeller bei Grundstücken ohne Braurecht sind durchschnittlich nur 14,9 Quadratmeter, die mit Braurecht durchschnittlich 24,2 Quadratmeter groß. Allerdings hatten die Brauberechtigten sehr unterschiedlich große Küchenkeller von 14 bis 38 Quadratmeter. Hier kommen unterschiedliche Nutzungsbedingungen zum Ausdruck. Die Besitzer großer Braugrundstücke verfügten häufig neben dem Braugewerbe, das ohnehin zumeist nur, wenn auch ein sehr einträglicher, Nebenerwerb war, noch über Handwerks- und Handelstätigkeit und Landwirtschaft, teilweise auch über Gastgebergewerbe. In zehn Fällen der vermessenen Kelleranlagen waren frühneuzeitliche Keller feststellbar. Sie sind mit durchschnittlich 7,4 Quadratmetern je Gebräude zehn Prozent größer als die Braukeller des Mittelalters.

Während die Denkmale der Stadt Torgau in einem Inventar von großer Vollständigkeit, soweit sie oberirdisch sind, erfasst und beschrieben worden sind, steht die Bestandsaufnahme für die unterirdischen Denkmale, die Keller, noch immer aus. ${ }^{22}$ Wir kennen noch nicht einmal ihre Anzahl und rechnen gegenwärtig mit etwa 300 Kelleranlagen (die aus mehreren Kellern bestehen) und Einzelkellern aus der Zeit vor 1800.

Diese Situation war für den Torgauer Geschichtsverein und die Stadt Torgau maßgebend, dem Tor-

\begin{tabular}{|c|c|c|c|c|}
\hline \multirow{2}{*}{$\begin{array}{c}\text { Gebräudeanzahl } \\
1514 / 25\end{array}$} & \multicolumn{3}{|c|}{ Bierkellergrößen je Gebräude in Quadratmeter } & \multirow{2}{*}{$\begin{array}{c}\text { Kellergröße } \\
\text { mittelalterlicher } \\
\text { Küchenkeller }\end{array}$} \\
\hline & $\begin{array}{l}\text { mittelalterlich } \\
\text { ohne } \\
\text { Küchenkeller }\end{array}$ & $\begin{array}{l}\text { mittelalterlich } \\
\text { mit } \\
\text { gesondertem } \\
\text { Küchenkeller }\end{array}$ & frühneuzeitlich & \\
\hline 3 & $11,2(3)$ & $8,2(7)$ & & $14,0(6)$ \\
\hline 4 & $11,0(4)$ & $8,5(1)$ & $9,4(2)$ & $14,7(2)$ \\
\hline 5 & $7,3(5)$ & $6,7(7)$ & & $29,6(7)$ \\
\hline 6 & $6,5(3)$ & $5,9(1)$ & $6,0(2)$ & $28,4(1)$ \\
\hline 7 & $7,0(6)$ & $6,3(5)$ & & $20,2(2)$ \\
\hline $8-9$ & $7,9(2)$ & $6,5(9)$ & $7,8(5)$ & 38,0 \\
\hline $10-12$ & -- & $5,3(7)$ & $6,7(1)$ & \\
\hline $\begin{array}{l}\text { durchschnittlich } \\
\text { ohne Braurecht }\end{array}$ & 8,5 & 6,8 & 7,4 & $\begin{array}{c}24,2 \\
14,9(8)\end{array}$ \\
\hline
\end{tabular}

Neben mittelalterlichen Einzelkellern, die sowohl als Braukeller aber auch gleichzeitig als Küchenkeller gedient haben, treten Kelleranlagen auf, die über Braukeller und gesonderte Küchenkeller verfügen. Die Küchenkeller waren häufig direkt von den Küchen aus erreichbar. In diesem Fall sind die ausschließlich als Braukeller genutzten durchschnittlich 20 Prozent kleiner und haben eine durchschnittliche Größe von 6,8 Quadratmetern je Gebräude. Ihre Gesamtgröße liegt je nach An- gauer Büro für Altbauforschung im Jahr 2016 den Auftrag zu erteilen, ein Kellerkataster für den Gesamtbestand zu erarbeiten. Für etwa 130 Grundstücke liegt eine Erfassung zwischenzeitlich vor. Der Abschluss des Projekts ist für 2019 vereinbart. Wir erwarten mit dem vorliegenden Gesamtkataster eine Grundlage besserer Kenntnisse über die Stadtentwicklung, aber auch über Nutzungsverhältnisse und nicht zuletzt über die Bierkeller zu gewinnen. 